\title{
Reverse Average Dwell-Times for Networked Control Systems
}

\author{
Stefan H.J. Heijmans
}

\author{
Romain Postoyan
}

\begin{abstract}
Most emulation-based results in networked control systems rely on a bound on the maximal allowable transmission interval (MATI) under which stability is preserved. However, having only such a MATI condition can lead to conservative results, as large values of transmission intervals may only occur sporadically, while the typical transmission interval is much smaller. In this paper, we therefore propose, in addition to the existence of a MATI, to also impose a bound on the average allowable transmission interval, expressed in terms of a reverse average dwell-time (RADT) condition on the transmission intervals. We provide joint conditions on the RADT and the MATI such that stability of the NCS can still be guaranteed, which can, in addition, lead to significant higher values of the MATI itself. The strengths of these new results are illustrated on a numerical example, showing a $484 \%$ improvement of the MATI, while still guaranteeing stability.
\end{abstract}

\section{INTRODUCTION}

Networked control systems (NCSs) are systems in which the sensors, controllers, and actuators of the plant are physically distributed and communicate via (packet-based) digital channels. This architecture is motivated by the many benefits it offers with respect to conventional (wired) control systems, including greater flexibility and low cost, see, e.g., [1]-[3]. However, inevitable network-induced imperfections such as varying transmission intervals due to, for instance, packet losses, varying delays, and communication constraints can all degrade the overall performance of the system. Moreover, as the communication network is often shared by multiple sensor, controller, and actuator nodes, there is a need for socalled scheduling protocols that govern the access of these nodes to the network.

To deal with these network-induced phenomena, a popular two-step design framework for NCSs is the so-called emulation-based method as advocated in [4] and [5] combined with Lyapunov-based analysis tools for hybrid systems, as reflected in, e.g., [6]-[16]. The idea is to first design a (robustly stabilizing) controller for the plant while ignoring the communication constraints. In the second step, conditions on the communication rate, e.g., bounds on the transmission intervals are provided to guarantee closed-loop stability and performance of the NCS. In fact, a considerable amount of

S.H.J. Heijmans and W.P.M.H. Heemels are with the Control Systems Technology Group of the Department of Mechanical Engineering, Eindhoven University of Technology, The Netherlands. Their work is supported by the Innovational Research Incentives Scheme under the VICI grant 'Wireless control systems: A new frontier in automation' (No. 11382), which is financed by the Netherlands Organization for Scientific Research (NWO).

R. Postoyan is with the Université de Lorraine, CNRS, CRAN, Nancy, France. His work is supported by HANDY project ANR-18-CE40-0010-02.

D. Nešić is with MIDAS Laboratory, the Department of Electrical and Electronic Engineering, the University of Melbourne, Australia. His work was supported under the Australian Research Council under the Discovery Project DP170104099.

E-mail corresponding author: s.h.j.heijmans@gmail.nl.

\author{
Dragan Nešić \\ W.P. Maurice H. Heemels
}

research has been focusing on determining the so-called maximal allowable transmission interval (MATI) for which the closed-loop NCS with the emulated controller is still stable, see, e.g., [4]-[14]. Hence, the problem of characterizing the MATI is instrumental in the analysis of NCSs.

However, a clear limitation of an analysis based only on a MATI condition is that the situation in which the length of each transmission interval is equal to this 'worst case' value (being the MATI bound) is allowed to occur and, therefore, needs to be covered in the analysis. Consequently, as this 'worst case' scenario is typically unrealistic to occur in practice, analyses from literature often lead to conservative bounds on the MATI. Indeed, we have in general varying transmission intervals due to various network effects implying that large transmission intervals close to the MATI bound may only occur sporadically, while the typical transmission interval is much smaller. It is, for instance, more likely to expect that situations occur at which the length of some transmission intervals is significantly larger than that of most transmission intervals (perhaps to cope with packet losses), provided that these 'longer' transmission intervals are somehow compensated for by a number of shorter intertransmission times to maintain stability of the NCS. Such a scenario is, for instance, motivated by task executions on general purpose multiprocessor platforms that exhibit variations in their execution times, see [17]. As this situation cannot be addressed by only using the concept of a MATI, there is a need for a greater modeling flexibility to include more specific information on the transmission intervals in the NCS model.

We therefore propose in this work an alternative condition on the transmission intervals to better capture their timevarying behavior. That is, in addition to the existence of a MATI, we also propose to impose a bound on the average allowable transmission interval, expressed in terms of a reverse average dwell-time (RADT) condition, as introduced in [18], [19] for hybrid systems, on the transmission instants. We will show that imposing such an additional RADT constraint on the transmission instants (cf. a bound on the average allowable transmission interval) leads to trade-offs between the RADT and the MATI, which will also be evident in the considered numerical example. In particular, it will follow that when we transmit faster on average (i.e., a lower value for the RADT), we can obtain significant larger values of the MATI with respect to the works of [8]-[10].

The remainder of this paper is organized as follows. After presenting the necessary preliminaries and definitions in Section II, the considered class of systems is described in Section III, including a suitable (extended) hybrid model for the NCS with the RADT constraint. In Section IV, we state our main 
result by providing explicit conditions on the RADT and the MAT such that stability of the NCS is guaranteed. Finally, in Section $\mathrm{V}$ we illustrate our results by means of a numerical example, showing that the MATI can indeed be significantly increased when having an additional RADT constraint, and in Section VI some concluding remarks are given.

\section{PRELiminaries}

The sets of real and natural numbers are denoted by $\mathbb{R}$ and $\mathbb{N}$, respectively, and the sets of non-negative real numbers and integers by $\mathbb{R}_{\geq 0}$ and $\mathbb{N}_{0}(:=\mathbb{N} \cup\{0\})$, respectively. For vectors $v_{1}, v_{2}, \ldots, v_{n} \in \mathbb{R}^{n}$, we denote by $\left(v_{1}, v_{2}, \ldots, v_{n}\right)$ the vector $\left[\begin{array}{cccc}v_{1}^{\top} & v_{2}^{\top} & \cdots & v_{n}^{\top}\end{array}\right]^{\top}$, and by $|\cdot|$ and $\langle\cdot, \cdot\rangle$ the Euclidean norm and the usual inner product, respectively. Moreover, we define the distance of a vector $x \in \mathbb{R}^{n}$ to a closed non-empty set $A \subset \mathbb{R}^{n}$ as $|x|_{A}:=\min _{y \in A}|x-y|$. The $n$ by $n$ identity and zero matrices are denoted by $I_{n}$ and $0_{n}$, respectively. For a symmetric matrix $A \in \mathbb{R}^{n \times n}, \lambda_{\max }(A)$ denotes the largest eigenvalue of $A$.

Consider the nonlinear hybrid system given by

$$
\mathcal{H}:\left\{\begin{aligned}
\dot{\xi} & =F(\xi), & \text { when } & \xi \in C \\
\xi^{+} & =G(\xi), & \text { when } & \xi \in D
\end{aligned}\right.
$$

where $\xi \in \mathbb{X} \subseteq \mathbb{R}^{m_{\xi}}$ is the state vector, $F$ the flow map, $G$ the jump map, $C \subseteq \mathbb{X}$ the flow set, $D \subseteq \mathbb{X}$ is the jump set, and the set of initial conditions is defined as $\mathbb{X}_{0} \subseteq C \cup D \subseteq \mathbb{X}$ (i.e., $\left.\xi(0,0) \in \mathbb{X}_{0}\right)$. We assume that the sets $C$ and $D$ are closed sets and that $F$ and $G$ are continuous.

A solution to a hybrid system of the form (1) is defined on a hybrid time domain, which is a subset of $\mathbb{R}_{\geq 0} \times \mathbb{N}_{0}$. In particular, consider the following definitions from [20].

Definition 1a: A subset $E \subset \mathbb{R}_{\geq 0} \times \mathbb{N}_{0}$ is a compact hybrid time domain if $E=\cup_{j=0}^{J-1}\left(\left[t_{j}, t_{j+1}\right] \times\{j\}\right)$ for some finite sequence of times $0=t_{0} \leq t_{1} \leq t_{2} \leq \ldots \leq t_{J}$.

Definition 1b: A hybrid time domain is any set $E \subset \mathbb{R}_{\geq 0} \times$ $\mathbb{N}_{0}$ such that for all $(T, J) \in E, E \cap([0, T] \times\{0,1, \ldots, J\})$ is a compact hybrid domain.

Definition 1c: A hybrid arc is a function $\xi$ defined on its hybrid time domain $\operatorname{dom} \xi$ that is for each $j \in \mathbb{N}_{0}$ locally absolutely continuous in t on the interval $(\operatorname{dom} \xi) \cap\left(\mathbb{R}_{\geq 0} \times\{j\}\right)$.

Definition 1d: A time instant $t \in \mathbb{R}_{\geq 0}$ is called a jump time for a hybrid arc $\xi$ if there is a $j \in \mathbb{N}$ such that $(t, j) \in$ $\operatorname{dom} \xi$ and $(t, j-1) \in \operatorname{dom} \xi$.

We will often not mention $\operatorname{dom} \xi$ explicitly, with the understanding that each hybrid arc $\xi$ is defined on its corresponding hybrid time domain $\operatorname{dom} \xi$. Moreover, observe that the sequence of times $0 \leq t_{1} \leq t_{2} \leq \ldots t_{J}$ as specified in Definition 1a for the hybrid time domain in Definition $1 \mathrm{~b}$ (with $t_{0}$ thus excluded) are jump times. Using these definitions, we say now that a hybrid arc $\xi \in \mathbb{X}$ is a solution to (1) with initial state set $\mathbb{X}_{0}$ if $\xi(0,0) \in \mathbb{X}_{0}$ and

- for all $j \in \mathbb{N}_{0}$ and almost all $t$ such that $(t, j) \in \operatorname{dom} \xi$, $\xi(t, j) \in C$ and $\dot{\xi}(t, j)=F(\xi(t, j))$

- for all $(t, j) \in \operatorname{dom} \xi$ such that $(t, j+1) \in \operatorname{dom} \xi$, $\xi(t, j) \in D$ and $\xi(t, j+1)=G(\xi(t, j))$.
Hence, $\xi(t, j+1)$ is denoted $\xi^{+}$in (1) and $\operatorname{dom} \xi$ denotes the hybrid time domain of $\xi$. In addition, a solution $\xi$ is called maximal, if there does not exist another solution $\tilde{\xi}$ such that $\operatorname{dom} \xi \subset \operatorname{dom} \tilde{\xi}$ and $\xi(t, j)=\tilde{\xi}(t, j)$ for all $(t, j) \in \operatorname{dom} \xi$, and that a solution $\xi$ is called $t$-complete if $\sup \left\{t \in \mathbb{R}_{\geq 0} \mid \exists j \in\right.$ $\mathbb{N}_{0}$ such that $\left.(t, j) \in \operatorname{dom} \xi\right\}=\infty$. For a detailed description and interpretation of these concepts, the interested reader is referred to [20].

We are interested in the stability of the hybrid system (1).

Definition 2: Consider the hybrid system $\mathcal{H}$ on $\mathbb{X}$ given by (1) and consider a closed set $\mathcal{E} \subseteq \mathbb{X}_{0}$. Then the set $\mathcal{E}$ is said to be uniformly globally exponentially stable (UGES) if there exist constants $K, c>0$ such that for any initial condition $\xi(0,0) \in \mathbb{X}_{0}$, all corresponding maximal solutions $\xi$ are $t$-complete and satisfy for all $(t, j) \in \operatorname{dom} \xi$

$$
|\xi(t, j)|_{\mathcal{E}} \leq K|\xi(0,0)|_{\mathcal{E}} e^{-c(t+j)} .
$$

\section{System SETUP}

In this section, we introduce the NCS setup, the RADT condition, and a hybrid model describing the overall dynamics including a new hybrid clock, which captures the RADT constraint on the transmission instants.

\section{A. Networked Control Configuration}

We consider the NCS setup as shown in Fig. 1, where the continuous-time plant $\mathcal{P}$ communicates with the controller $\mathcal{C}$ via the network $\mathcal{N}$. We assume here that the controller is designed while ignoring the network, which is characteristic for the emulation-based design approach.

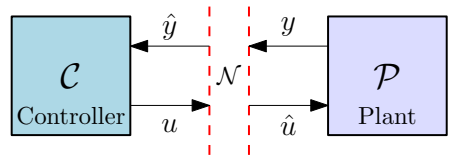

Fig. 1. The NCS setup as described in [4]-[14].

The plant and controller dynamics are given by

$$
\mathcal{P}:\left\{\begin{aligned}
\dot{x}_{p} & =f_{p}\left(x_{p}, \hat{u}\right) \\
y & =g_{p}\left(x_{p}\right)
\end{aligned} \quad \text { and } \mathcal{C}:\left\{\begin{aligned}
\dot{x}_{c} & =f_{c}\left(x_{c}, \hat{y}\right) \\
u & =g_{c}\left(x_{c}\right),
\end{aligned}\right.\right.
$$

where $x_{p} \in \mathbb{R}^{m_{x_{p}}}$ and $x_{c} \in \mathbb{R}^{m_{x_{c}}}$ denote the plant and controller state, respectively, $u \in \mathbb{R}^{m_{u}}$ the control input, $\hat{u} \in \mathbb{R}^{m_{u}}$ the most recently received control input by the plant, $y \in \mathbb{R}^{m_{y}}$ the output, and $\hat{y} \in \mathbb{R}^{m_{y}}$ the most recently received output of the plant. We assume that $f_{p}$ and $f_{c}$ are continuous, and $g_{p}$ and $g_{c}$ continuously differentiable.

To complete the NCS setup, we also need to describe the network $\mathcal{N}$ in more detail. As such, we assume that it has a collection of sampling/transmission times $t_{j}, j \in \mathbb{N}$, which satisfy $0 \leq t_{1}<t_{2}<\ldots$. At such a time $t_{j}$, (parts of) the output $y$ and the input $u$ are sampled and transmitted to the controller $\mathcal{C}$ and the plant $\mathcal{P}$, respectively, which results in an update of (a part of) the networked values according to

$$
\begin{aligned}
& \hat{y}\left(t_{j}^{+}\right)=y\left(t_{j}\right)+h_{y}\left(j, e\left(t_{j}\right)\right) \\
& \hat{u}\left(t_{j}^{+}\right)=u\left(t_{j}\right)+h_{u}\left(j, e\left(t_{j}\right)\right),
\end{aligned}
$$


where the function $h:=\left(h_{y}, h_{u}\right)$ with $h: \mathbb{N}_{0} \times \mathbb{R}^{m_{e}} \rightarrow$ $\mathbb{R}^{m_{e}}$ models the scheduling protocol that determines which (sensor and/or actuator) node is granted access to the network at time $t_{j}$, see, e.g., [6]-[8], and where $e$ denotes the network-induced error defined by $e:=\left(e_{y}, e_{u}\right)=(\hat{y}-y, \hat{u}-u)$. We also assume that $\hat{y}$ and $\hat{u}$ are constant in between two successive transmissions (i.e., the network nodes operate in a similar manner to a zero-order-hold ( $\mathrm{ZOH})$ ). However, this can easily be modified, if desired, see [6].

Finally, as shown by the works of, e.g., [4]-[14], it is common to assume that (all of) the transmission intervals are bounded by

$$
\tau_{\text {miati }} \leq t_{j+1}-t_{j} \leq \tau_{\text {mati }}, \quad j \in \mathbb{N},
$$

where $\tau_{\text {mati }}$ denotes the maximal allowable transmission interval (MATI) and $\tau_{\text {miati }}$ the minimal allowable transmission interval (MIATI), such that $0<\tau_{\text {miati }} \leq \tau_{\text {mati }}$. The upper bound $\tau_{\text {mati }}$ is used in many papers to guarantee stability properties of the NCS designed using the emulation-based approach, see, e.g., [7]-[14], while the lower bound on the transmission intervals $\tau_{\text {miati }}>0$ is often taken arbitrarily small, see, e.g., [4]-[8], [11]-[14], since it is only imposed to prevent Zeno behavior. However, note that in practice this lower bound always exists due to limitations in digital communication hardware in terms of a smallest achievable transmission period. In fact, knowledge of the MIATI (instead of assuming it to be arbitrarily small) can be even exploited to improve the MATI as was recently shown in [9] and [10] and as will also follow from our analysis (see Remark 4).

Combining the continuous-time behavior of the physical system (2) and the discrete-event behavior of the network (3), the NCS setup described by (2)-(4) can be written in a hybrid system formalism as advocated in [6] and described in [8], which is of the form of (1) where each 'jump' of the hybrid system corresponds to an update of the networked values, i.e., the sequence of transmission times of the NCS is equal to the sequence of jump times of the hybrid model. Using this hybrid modeling framework, stability and performance of the NCS were analyzed in [7]-[14] using Lyapunovbased techniques for hybrid systems, resulting in bounds on the MATI such that, for instance, UGES for the NCS is guaranteed.

Condition (4), however, allows for all of the transmission intervals to have a length equal to the MATI ('worst case'), which, as mentioned in the introduction, is often unrealistic in practice and conservative in the analysis. As such, our objective in this work is to include more specific information concerning the transmission intervals in the NCS model in terms of an average allowable transmission interval ('average case'). In particular, we propose an extended hybrid model for the NCS that captures a RADT condition on the jump times of the hybrid system (cf. transmission instants), in addition to the MIATI and MATI constraints as in (4). It will follow that incorporating information on the average transmission interval (through a RADT condition) results in significantly larger values for the MATI compared to [8], [9] or [10].

\section{B. A RADT Augmented Hybrid Model for the NCS}

As introduced in [18], [19] for hybrid systems, a RADT condition on the transmission instants for NCSs translates to requiring that, on average, at least one transmission on the networked values occurs in an interval of length $\tau_{r-d t}^{*}$ called the reverse average dwell-time (RADT). To describe a NCS setup with RADT condition next to the MIATI and MATI constraints as in (4), we propose in this work, based on [6] and [8], an extended hybrid system $\mathcal{H}_{n c s}$, which requires the introduction of a new 'clock' variable as the existing hybrid clocks from literature, see [6], [8], [22]-[24], do not incorporate the MIATI, the MATI and the RADT constraint simultaneously. In particular, we consider the hybrid system as given by

$$
\mathcal{H}_{n c s}:\left\{\begin{array}{rlll}
\dot{\xi} & =F(\xi) & \text { when } & \tau \in\left[0, \tau_{\text {mati }}\right] \\
\xi^{+}=G(\xi) & \text { when } & \tau \in\left[s+\tau_{\text {miati }}, \tau_{\text {mati }}\right]
\end{array}\right.
$$

with its full state $\xi:=\left(\left(x_{p}, x_{c}\right), e,(\tau, s), \kappa\right) \in \mathbb{X}:=\mathbb{R}^{m_{x}} \times$ $\mathbb{R}^{m_{e}} \times\left[0, \tau_{\text {mati }}\right] \times \mathbb{R} \times \mathbb{N}_{0}$ and the set of initial conditions

$$
\mathbb{X}_{0}:=\mathbb{R}^{m_{x}} \times \mathbb{R}^{m_{e}} \times\left[0, \tau_{\text {mati }}\right] \times\left(-\infty, \tau_{\text {mati }}-\tau_{\text {miati }}\right] \times \mathbb{N}_{0},
$$

and where, for the appropriately chosen RADT $\tau_{r-d t}^{*}>0$ such that $0<\tau_{\text {miati }} \leq \tau_{r-d t}^{*} \leq \tau_{\text {mati }}, F(\xi):=(f(x, e), g(x, e)$, $1,0,0)$ and $G(\xi):=\left(x, h(\kappa, e), \max \left\{0, \tau-\tau_{r-d t}^{*}\right\}, \max \{0\right.$, $\left.\left.\tau-\tau_{r-d t}^{*}\right\}, \kappa+1\right)$ with $x:=\left(x_{p}, x_{c}\right) \in \mathbb{R}^{m_{x}}, m_{x}=m_{x_{p}}+m_{x_{c}}$, and

$$
\begin{aligned}
f(x, e): & :=\left[\begin{array}{l}
f_{p}\left(x_{p}, g_{c}\left(x_{c}\right)+e_{u}\right) \\
f_{c}\left(x_{c}, g_{p}\left(x_{p}\right)+e_{y}\right)
\end{array}\right] \\
g(x, e) & :=\left[\begin{array}{l}
-\frac{\partial g_{p}}{\partial x_{p}} f_{p}\left(x_{p}, g_{c}\left(x_{c}\right)+e_{u}\right) \\
-\frac{\partial g_{c}}{\partial x_{c}} f_{c}\left(x_{c}, g_{p}\left(x_{p}\right)+e_{y}\right)
\end{array}\right] .
\end{aligned}
$$

Here, $\kappa \in \mathbb{N}_{0}$ is, similar to [6], [8], a counter variable (which keeps track of the amount of transmissions) and $\tau \in \mathbb{R}_{\geq 0}$ a timer variable, while $s \in \mathbb{R}$ is a newly introduced memory variable initialized at a value $s(0,0) \leq \tau_{\text {mati }}-\tau_{\text {miati }}$ and used to 'remember' to which value $\tau$ was reset after a jump of the hybrid system $\mathcal{H}_{n c s}$ has occurred. As such, the pair of variables $(\tau, s)$ comprises now a novel 'hybrid clock' that captures the MIATI, MATI, and RADT constraints on the jump times/transmission instants in a hybrid model. For more details concerning the hybrid model (5) we refer to [21]. However, it is important to observe that for the hybrid system $\mathcal{H}_{n c s}$ we have the following result.

Proposition 1: Let $0<\tau_{\text {miati }} \leq \tau_{r-d t}^{*} \leq \tau_{\text {mati }}$ be given. A hybrid time domain $E$ with its sequence of jump times $\left\{t_{j}\right\}_{j \in \mathbb{N}}$, satisfies

$$
\begin{aligned}
t_{j+1}-t_{j} \geq \tau_{\text {miati }} & \text { for all } j \in \mathbb{N} \\
j-i \geq \frac{(\bar{t}-t)-\tau_{\text {mati }}}{\tau_{r-d t}^{*}} & \text { for all }(t, i),(\bar{t}, j) \in E \\
& \text { with } \bar{t}+j \geq t+i
\end{aligned}
$$

if and only if $E=\operatorname{dom} \xi$ for some solution $\xi$ to (5) with initial state set $\mathbb{X}_{0}$ given by (6).

The proof can be found in [21]. Condition (7a) corresponds to two consecutive jumps of the hybrid system (5) being sep- 
arated by at least $\tau_{\text {miati }}$ time units, and thus that each transmission interval of the NCS modeled by the hybrid system (5) has a lower bound equal to the MIATI. Condition (7b), on the other hand, corresponds to the RADT constraint on the transmission instants as introduced above and indicates that there are at most $\tau_{\text {mati }}$ time units between two consecutive transmissions (cf. all the transmission intervals are upper bounded by the MATI), see [21]. Proposition 1 thus shows that the hybrid model (5) for a NCS does not only include a MIATI and a MATI bound on the transmission intervals, but also captures a RADT. Using this augmented hybrid system (5) (including average transmission interval conditions), we will analyze for which triples of $\left(\tau_{\text {miati }}, \tau_{r-d t}^{*}, \tau_{\text {mati }}\right)$ the set

$$
\mathcal{E}:=\{\xi \in \mathbb{X} \mid x=0 \text { and } e=0\}
$$

is guaranteed to be UGES for the NCS.

Remark 1: In the special case of $\tau_{r-d t}^{*}=\tau_{\text {mati }},(7 \mathrm{~b})$ actually recovers a MATI-like bound as imposed by (the right hand-side of) the 'classical' condition (4), i.e., in the case of $\tau_{r-d t}^{*}=\tau_{\text {mati }}$, the $R A D T$ condition $(7 \mathrm{~b})$ requires that two consecutive transmission times must be separated by at most $\tau_{r-d t}^{*}\left(=\tau_{\text {mati }}\right)$ time units.

Remark 2: Condition (7b) also relates the values for $\tau_{r-d t}^{*}$ and $\tau_{\text {mati }}$ to each other. That is, when we on average transmit slightly faster/slower (take $\tau_{r-d t}^{*}$ lower/higher), then $\tau_{\text {mati }}$ can be larger/smaller. This trade-off between the values for the RADT and the MATI will also be evident from our Lyapunov-based analysis, which follows below.

\section{Stability ANALYSIS}

Here, we directly provide conditions on the RADT and the MATI such that UGES of the set $\mathcal{E}$ is guaranteed. To do so, in line with [6], [8], [11], we first make the following assumption.

Assumption 1: Consider the hybrid system $\mathcal{H}_{n c s}$ as in (5). There exist a function $W: \mathbb{N}_{0} \times \mathbb{R}^{m_{e}} \rightarrow \mathbb{R}_{\geq 0}$ that is locally Lipschitz in its second argument, a locally Lipschitz function $V: \mathbb{R}^{m_{x}} \rightarrow \mathbb{R}_{\geq 0}$, a continuous function $H: \mathbb{R}^{m_{x}} \rightarrow \mathbb{R}$, and constants $\lambda \in(0,1), L \geq 0, \underline{\alpha}_{W}, \bar{\alpha}_{W}, \underline{\alpha}_{V}, \bar{\alpha}_{V}, \varepsilon, \gamma>0$ such that the following hold:

1) For all $\kappa \in \mathbb{N}_{0}$ and $e \in \mathbb{R}^{m_{e}}$

$$
\begin{aligned}
\underline{\alpha}_{W}|e| \leq W(\kappa, e) & \leq \bar{\alpha}_{W}|e| \\
W(\kappa+1, h(\kappa, e)) & \leq \lambda W(\kappa, e) .
\end{aligned}
$$

2) For all $\kappa \in \mathbb{N}_{0}, x \in \mathbb{R}^{m_{x}}$, and almost all $e \in \mathbb{R}^{m_{e}}$

$$
\left|\frac{\partial W(\kappa, e)}{\partial e}, g(x, e)\right| \leq L W(\kappa, e)+H(x) .
$$

3) For all $x \in \mathbb{R}^{m_{x}}$

$$
\underline{\alpha}_{V}|x|^{2} \leq V(x) \leq \bar{\alpha}_{V}|x|^{2} .
$$

4) For all $\kappa \in \mathbb{N}_{0}$, $e \in \mathbb{R}^{m_{e}}$, and almost all $x \in \mathbb{R}^{m_{x}}$

$$
\langle\nabla V(x), f(x, e)\rangle \leq-\varepsilon^{2} V(x)-H^{2}(x)+\gamma^{2} W^{2}(\kappa, e) .
$$

This assumption is essentially the same as the main assumption [8, Assumption 1], see also [21]. Moreover, these conditions (9)-(12) have been proven to be systematically checkable for various classes of NCSs, see, e.g., [7]-[14].

Now, based on extensions of the stability results for networked control systems as in [8]-[10], novel conditions under which the set $\mathcal{E}$ is UGES can be obtained.

Theorem 1: Under Assumption 1 and for a given value of the MIATI $\tau_{\text {miati }}>0$, if the RADT $\tau_{r-d t}^{*} \geq \tau_{\text {miati }}$ satisfies for some constants $0<\delta<\varepsilon^{2}$ and $\psi:=2 L+\varepsilon^{2}$ the bound

$$
\tau_{\mathrm{r}-\mathrm{dt}}^{*}<\left|\frac{\ln \left(\lambda^{2}\right)-\left(\varepsilon^{2}-\delta\right) \tau_{\text {miati }}}{2 \gamma+\psi}\right|,
$$

and if the MATI $\tau_{m a t i} \geq \tau_{r-d t}^{*}$ satisfies the bound $\tau_{m a t i} \leq$ $T\left(\lambda, \tau_{r-d t}^{*}\right)$, where

$T\left(\lambda, \tau_{r-d t}^{*}\right)= \begin{cases}\frac{2}{\psi r} \arctan \left(\frac{r(1-\sigma)}{2 \frac{\sigma}{1+\sigma}\left(\frac{2 \gamma}{\psi}-1\right)+1+\sigma}\right), & 2 \gamma>\psi \\ \frac{2}{\psi} \frac{1-\sigma}{1+\sigma}, & 2 \gamma=\psi \\ \frac{2}{\psi r} \operatorname{arctanh}\left(\frac{r(1-\sigma)}{2 \frac{\sigma}{1+\sigma}\left(\frac{2 \gamma}{\psi}-1\right)+1+\sigma}\right), & 2 \gamma<\psi\end{cases}$

with $\sigma:=-\frac{1}{2 \gamma}\left(\sqrt{\left(\psi+\lambda_{W}\right)^{2}-4 \gamma^{2}}+\left(\psi+\lambda_{W}\right)\right)>0, \lambda_{W}:=$ $\frac{\ln \left(\lambda^{2}\right)-\left(\varepsilon^{2}-\delta\right) \tau_{m i a t i}}{\tau_{r-d t}^{*}}$, and $r:=\sqrt{\left|\left(\frac{2 \gamma}{\psi}\right)^{2}-1\right|}$, then the set $\mathcal{E}$ given by (8) is UGES for the hybrid system (5).

The full proof is provided in [21] for the reader's convenience, which is based on the construction of a genuine (hybrid) Lyapunov function in the sense that it decreases during flows (cf. in between transmission instants) and does not increase during jumps (cf. when a transmission on the networked value occurs) of the hybrid system to show stability. In particular, in view of Assumption 1 and inspired by [8], [9] and [10], the hybrid Lyapunov function

$$
U(\xi)=\phi_{V}(\tau) V(x)+\gamma \phi_{W}(\tau) W^{2}(\kappa, e)
$$

is proposed where $\phi_{V}:\left[0, \tau_{\text {mati }}\right] \rightarrow \mathbb{R}_{\geq 0}$ is an exponentially increasing function and $\phi_{W}:\left[0, \tau_{\text {mati }}\right] \rightarrow \mathbb{R}_{\geq 0}$ a strictly decreasing function, both designed such that $U$ indeed is strictly decreasing during flows, see [21] for the details. Consequently, it is shown under which conditions on the functions $\phi_{V}$ and $\phi_{W}$, and the transmission interval bounds $\tau_{r-d t}^{*}$ and $\tau_{m a t i}$ the Lyapunov function $U$ given by (15) also satisfy its jump condition. Here, however, it should be observed that, as the function $\phi_{V}$ is increasing, the function $\phi_{W}$ is decreasing, and given the fact that $\tau$ 'jumps' to a lower value at an update of the networked values (see (5)), the property of $U$ satisfying its jump condition is directly related to the amount the function $\phi_{W}$ decreases in value over a time-interval equal to $\tau_{r-d t}^{*}$. That is, when the decrease rate (i.e., derivative) of the function $\phi_{W}$ exceeds a certain (timer-dependent) threshold value, the Lyapunov function $U$ given by (15) fails to satisfy its jump condition. As such, it follows that, for a given value of the RADT $\tau_{r-d t}^{*}$, the value for the MATI $\tau_{\text {mati }}$ can be determined as being the 
point in time at which the decrease rate of the function $\phi_{W}$ attains this threshold value. To this end, the derivative of the function $\phi_{W}$ is bounded from below and it is verified when this bound fails to hold, which leads to the explicit solution for the MATI given by (14).

Based on the bounds presented in Theorem 1, it follows that the value for the MATI $\tau_{\text {mati }}$ indeed depends on the value for the RADT $\tau_{r-d t}^{*}$ through $\lambda_{W}$. In particular, the value for $\tau_{\text {mati }}$ increases when the value for $\left|\lambda_{W}\right|$ increases (as the value for $\sigma$ decreases in this case, i.e., becomes closer to 0 ), which corresponds to having a smaller value for $\tau_{r-d t}^{*}$. As such, smaller values of $\tau_{r-d t}^{*}>\tau_{\text {miati }}$ will results in higher values for the MATI, while for increasing values of $\tau_{r-d t}^{*}$ we have that $\tau_{\text {mati }}$ becomes smaller. This trade-off between the values for the RADT and the MATI will also be illustrated on a numerical example in the Section V.

Remark 3: To obtain the highest possible value for $\tau_{\text {mati }}$, we will take, in practice, $\delta>0$ as small as possible (i.e., essentially zero) since this results in the highest possible value for $\left|\lambda_{W}\right|$, which is desired, see [21]. Moreover, it follows from both the bounds (13) and (14) for $\tau_{r-d t}^{*}$ and $\tau_{\text {mati }}$, respectively, that they are maximal when $\gamma$ is taken as small as possible (while satisfying (12)). Similarly, it also follows that having $\varepsilon$ large would in general lead to higher values of the RADT and the MATI, especially when the value for the MIATI is large. However, this would also lead to the value of $\gamma$ being larger due to constraint (12), which results in lower values for the RADT and the MATI. Therefore, we will in general take $\varepsilon$ small, however, it is this balancing effect that might give in some specific cases better results for larger values of $\varepsilon$.

Remark 4: Hypothetically, having $\tau_{\text {miati }}=0$ in the value for $\lambda_{W}$ would already be sufficient for Theorem 1 to result in feasible values for $\tau_{r-d t}^{*}$ and $\tau_{\text {mati }}$. As such and since for larger values of $\tau_{\text {miati }}>0$ the value for $\left|\lambda_{W}\right|$ becomes larger, it directly follows, similar to [9], [10], that exploiting knowledge on the existence of a positive MIATI leads to higher values for the MATI (when $\delta<\varepsilon^{2}$ ).

\section{Numerical EXAMPLE}

To illustrate the application of our results and to make a comparison with the results of [8]-[10], we consider the same numerical example as in [9], [10] of stabilizing an open-loop unstable plant $\mathcal{P}$ with a state-feedback controller $\mathcal{C}$, which are modeled by

$$
\mathcal{P}: \dot{x}_{p}=A_{\mathbf{P}} x_{p}+B_{\mathbf{P}} u \text { and } \mathcal{C}: u=-K \hat{x}_{p}
$$

with, respectively, $\hat{x}_{p}$ the networked value of the plant state $x_{p}$ and

$A_{\mathbf{P}}=\frac{1}{5}\left(\begin{array}{ll}-4 & 1 \\ -2 & 3\end{array}\right), \quad B_{\mathbf{P}}=\left(\begin{array}{c}-1 \\ 2\end{array}\right), \quad$ and $\quad K=\left(\begin{array}{ll}-0.2 & 0.5\end{array}\right)$.

Hence, from this system configuration it thus follows that we assume that the plant states $x_{p}$ are transmitted over a network
$\mathcal{N}$ to the controller, i.e., we have a NCS with the networkinduced error given by $e=\hat{x}_{p}-x_{p}$. Moreover, we consider the case with the number of nodes in the network being $\ell=2$. As a result, we can model the closed-loop dynamics as a hybrid system of the form (5) where $f(x, e)=\mathbf{A} x+\mathbf{E} e$ and $g(x, e)=\mathbf{C} x+\mathbf{F} e$ with $\mathbf{A}:=A_{\mathbf{P}}-B_{\mathbf{P}} K, \mathbf{E}:=-B_{\mathbf{P}} K$, $\mathbf{C}:=-\mathbf{A}$, and $\mathbf{F}:=-\mathbf{E}$.

For this linear example, the conditions (10)-(12) reduce to a single linear matrix inequality (LMI) condition when we assume that for some constant $M>0$, almost all $e \in \mathbb{R}^{m_{e}}$, and for all $\kappa \in \mathbb{N}_{0}$ it holds that $\left|\frac{\partial W(\kappa, e)}{\partial e}\right| \leq M$, see, e.g., [9]. In particular, conditions (10)-(12) can be summarized into the LMI condition

$$
\left[\begin{array}{cc}
\mathbf{A}^{\top} X_{\mathbf{T}}+X_{\mathbf{T}} \mathbf{A}+\varepsilon^{2} X_{\mathbf{T}}+M^{2} \mathbf{C}^{\top} \mathbf{C} & X_{\mathbf{T}} \mathbf{E} \\
\mathbf{E}^{\top} X_{\mathbf{T}} & -\left(\underline{\alpha}_{W}\right)^{2} \gamma^{2} I_{m_{e}}
\end{array}\right] \leq 0,
$$

where we have chosen $V(x)=x^{\top} X_{\mathbf{T}} x$ with $X_{\mathbf{T}}$ being a symmetric positive definite matrix of size $m_{x} \times m_{x}$ such that $\underline{\alpha}_{V}=\lambda_{\min }\left(X_{\mathbf{T}}\right), \bar{\alpha}_{V}=\lambda_{\max }\left(X_{\mathbf{T}}\right), L=M \underline{\alpha}_{W}^{-1}|\mathbf{F}|$ and $H(x)=M|\mathbf{C} x|$, see, for instance, [9, Section V].

For a given scheduling protocol, in this example the TOD protocol with $\lambda=\sqrt{\ell-1 / \ell}=\frac{1}{2} \sqrt{2}$ and $\underline{\alpha}_{W}=\bar{\alpha}_{W}=M=1$, we can now use the LMI condition (17) in combination with Theorem 1 to compute the values for the pair $\left(\tau_{r-d t}^{*}, \tau_{m a t i}\right)$ such that UGES for the NCS described by (16) is guaranteed. The results for $\tau_{\text {miati }}=10^{-3}$ are given in Fig. 2, along with the obtained value for the MATI using [8, Theorem 1].

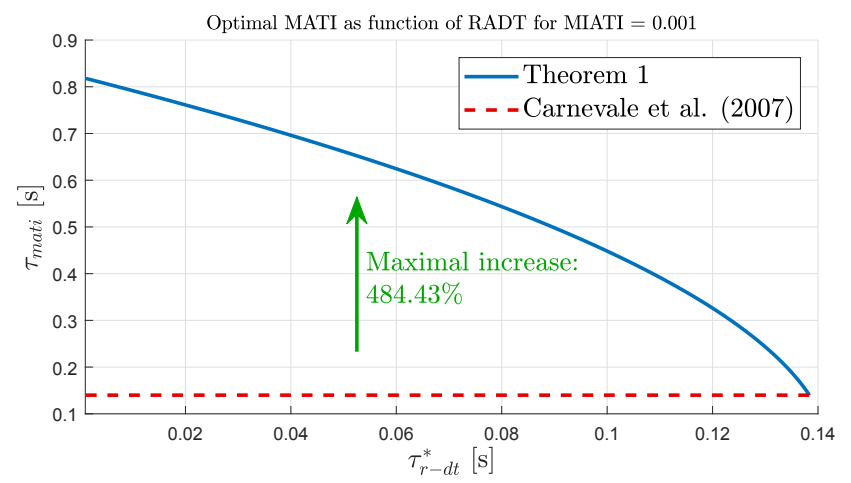

Fig. 2. Computed values for $\left(\tau_{r-d t}^{*}, \tau_{\text {mati }}\right)$ using Theorem 1 for $\tau_{\text {miati }}=$ 0.001 and the TOD protocol. Also the computed value for the MATI from [8] is indicated in the figure, along with the maximal attained improvement with respect to [8].

As shown by Fig. 2, there indeed is a clear trade-off between the RADT and the MATI as discussed earlier in Remark 2. That is, higher values of $\tau_{r-d t}^{*}$ indeed lead to lower values of $\tau_{m a t i}$. Moreover, for $\tau_{r-d t}^{*}=\tau_{\text {mati }}$ (i.e., the case that the RADT actually represents a MATI bound, see also Remark 2), we obtain a good approximation of the obtained value for the MATI as in [8].

To make now a clearer comparison with the results from [8]-[10], some of the results from Fig. 2 have been singled out in Table I. Here $\tau_{m a t i}^{[8]}$ represents the value obtained using the results from [8], $\tau_{\text {mati }}^{[9]}$ the value obtained using the result from [9] (where $\tau_{\text {miati }}=\tau_{\text {mati }}^{[8]}$ has been used in 
TABLE I

THE BOUND ON THE MATI FOR $\tau_{\text {miati }}=10^{-3}$ WITH $L=1.204, \lambda=\frac{1}{2} \sqrt{2}, \delta=10^{-6}, \varepsilon=0.1$, AND $\gamma=1.222$.

\begin{tabular}{|c|c|c|c|c|c|c|c|}
\hline \multirow[b]{2}{*}{$\tau_{m a t i}^{[8]}$} & \multirow{2}{*}{$\tau_{\text {mati }}^{[9]}$} & \multirow{2}{*}{$\tau_{\text {mati }}^{[10]}$} & \multicolumn{4}{|c|}{$\tau_{\text {mati }}$} & \multirow{2}{*}{$\begin{array}{c}\text { Maximal attained } \\
\text { improvement } \\
\text { of } \tau_{\text {mati }} \text { w.r.t. } \tau_{\text {mati }}^{[8]}\end{array}$} \\
\hline & & & $\tau_{r-d t}^{*}<0.137$ & $\tau_{r-d t}^{*}<0.109$ & $\tau_{r-d t}^{*}<0.020$ & $\tau_{r-d t}^{*}<0.0011$ & \\
\hline 0.13993 & 0.14009 & 0.14017 & 0.16157 & 0.39793 & 0.76112 & 0.81782 & $484.43 \%$ \\
\hline
\end{tabular}

the computations), $\tau_{m a t i}^{[10]}$ the value obtained using the result from [10], and $\tau_{\text {mati }}$ the value computed using Theorem 1. It can be directly seen that exploiting a RADT condition leads to significantly larger values for the MATI. Stated differently, information about the average transmission interval case can be exploited to allow much larger values of the 'worst case' transmission interval, while still guaranteeing stability. That is, when we have, for instance, on average a slightly smaller value for the transmission intervals than the MATI from [8] (e.g., $\tau_{r-d t}^{*}<0.137<\tau_{\text {mati }}^{[8]}$ ), we can obtain quite an extensive improvement of the maximal allowable transmission interval $\tau_{\text {mati }}$ (e.g., an improvement of $15.46 \%$ in the case of $\tau_{r-d t}^{*}<0.137$ or even an improvement of $484.43 \%$ in the case of $\tau_{r-d t}^{*}<0.0011$ with respect to the results in [8]). As such, when a RADT is exploited, the time between two transmissions is allowed to be significantly larger than the computed MATI as in [8] (or [9], [10]).

\section{CONCLUSION}

In this paper, we proposed a richer hybrid model description for NCSs designed using the emulation-based approach by including, next to the presence of a minimal and maximal allowable transmission interval (MIATI/MATI), the specification of a bound on the average allowable transmission interval given by means of a reverse average dwell-time (RADT) condition. We provided conditions on the RADT and the MATI such that UGES for the NCS is guaranteed and showed that there exists a trade-off between these values. In particular, it follows that transmitting on average faster (smaller RADT), directly leads to higher values for the MATI. We showed for a numerical example with the TOD protocol that improvements of the MATI up to $484 \%$ could be obtained with respect to the existing results of [8].

\section{REFERENCES}

[1] T.C. Yang, "Networked control system: A brief survey," IEE Proceedings Control Theory and Applications, vol. 153, no. 4, p. 403, 2006.

[2] J.P. Hespanha, P. Naghshtabrizi, and Y. Xu, "A survey of recent results in networked control systems," Proceedings of the IEEE, vol. 95, no. 1, pp. 138-162, 2007.

[3] L. Hetel, C. Fiter, H. Omran, A. Seuret, E. Fridman, J. Richard, and S.I. Niculescu, "Recent developments on the stability of systems with aperiodic sampling: An overview," Automatica, vol. 76, pp. 309-335, 2017.

[4] G.C. Walsh, O. Beldiman, and L.G. Bushnell, "Asymptotic behavior of nonlinear networked control systems," IEEE Transactions on Automatic Control, vol. 46, no. 7, pp. 1093-1097, 2001.

[5] G.C. Walsh, H. Ye, and L.G. Bushnell, "Stability analysis of networked control systems," IEEE Transactions on Control Systems Technology, vol. 10, no. 3, pp. 438-446, 2002.

[6] D. Nešić and A.R. Teel, "Input-output stability properties of networked control systems," IEEE Transactions on Automatic Control, vol. 49, no. 10, pp. 1650-1667, 2004.
[7] W.P.M.H. Heemels, A.T. Teel, N. van de Wouw, and D. Nešić, "Networked control systems with communication constraints: Tradeoffs between transmission intervals, delays, and performance," IEEE Transactions on Automatic Control, vol. 55, no. 8, pp. 1781-1796, 2010.

[8] D. Carnevale, A.R. Teel, and D. Nešić, “A Lyapunov proof of an improved maximum allowable transfer interval for networked control systems," IEEE Transactions on Automatic Control, vol. 52, no. 5, pp. 892-897, 2007.

[9] S.H.J. Heijmans, R. Postoyan, D. Nešić, and W.P.M.H. Heemels, "Computing minimal and maximal allowable transmission intervals for networked control systems using the hybrid systems approach," IEEE Control Systems Letters, vol. 1, no. 1, pp. 56-61, 2017.

[10] S.H.J. Heijmans, D. Nešić, R. Postoyan, and W.P.M.H. Heemels, "A generalized hybrid Lyapunov proof for networked control systems: Improving the maximum allowable transmission interval," in Proceedings of the IEEE $57^{\text {th }}$ Conference on Decision and Control, 2018, pp. 428433.

[11] D. Nešić, A.R. Teel, and D. Carnevale, "Explicit computation of the sampling period in emulation of controllers for nonlinear sampled-data systems," IEEE Transactions on Automatic Control, vol. 54, no. 3, pp. 619-624, 2009.

[12] M. Tabbara, D. Nešić, and A.R. Teel, "Stability of wireless and wireline networked control systems," IEEE Transactions on Automatic Control, vol. 52, no. 9, pp. 1615-1630, 2007.

[13] T. Ahmed-Ali and F. Lamnabhi-Lagarrigue, "High gain observer design for some networked control systems," IEEE Transactions on Automatic Control, vol. 57, no. 4, pp. 995-1000, 2012.

[14] K.Z. Liu, R. Wang, and G.P. Liu, "Tradeoffs between transmission intervals and delays for decentralized networked control systems based on a gain assignment approach," IEEE Transactions on Circuits and Systems II: Express Briefs, vol. 63, no. 5, pp. 498-502, 2016.

[15] F. Ferrante, F. Gouaisbaut, R.G. Sanfelice, and S. Tarbouriech, "State estimation of linear systems in the presence of sporadic measurements," Automatica, vol. 73, pp. 101-109, 2016.

[16] Y. Li, S. Phillips, and R.G. Sanfelice, "Robust distributed estimation for linear systems under intermittent information," IEEE Transactions on Automatic Control, vol. 63, no. 4, pp. 973-988, 2018.

[17] S. Adyanthaya, Z. Zhang, M. Geilen, J. Voeten, T. Basten, and R. Schiffelers, "Robustness analysis of multiprocessor schedules," in Proceedings of the International Conference on the Embedded Computer Systems: Architectures, Modeling, and Simulation (SAMOS XIV), 2014, pp. 9-17.

[18] J.P. Hespanha, D. Liberzon, and A.R. Teel, "On input-to-state stability of impulsive systems," in Proceedings of the IEEE $44^{\text {th }}$ Conference on Decision and Control, 2005, pp. 3992-3997.

[19] — - "Lyapunov conditions for input-to-state stability of impulsive systems," Automatica, vol. 44, no. 11, pp. 2735-2744, 2008.

[20] R. Goebel, R.G. Sanfelice, and A.R. Teel, Hybrid dynamical systems. United Kingdom: Princeton University Press, 2012.

[21] S.H.J. Heijmans, R. Postoyan, D. Nešić, and W.P.M.H. Heemels, "An average allowable transmission interval condition for the stability of metworked control systems," 2019, submitted for publication.

[22] D. Nešić and A.R. Teel, "A Lyapunov-based small-gain theorem for hybrid ISS systems," in Proceedings of the 47th IEEE Conference on Decision and Control, 2008, pp. 3380-3385.

[23] D. Nešić, A.R. Teel, G. Valmorbida, and L. Zaccarian, "Finite-gain $\mathcal{L}_{p}$-stability for hybrid dynamical systems," Automatica, vol. 49 , no. 8 , pp. 2384-2396, 2013.

[24] C. Cai, A.R. Teel, and R. Goebel, "Smooth Lyapunov functions for hybrid systems part II: (pre-)Asymptotically stable compact sets," IEEE Transactions on Automatic Control, vol. 53, no. 3, pp. 734-748, 2008. 


\section{University Library}

\section{- M M N E R VA A gateway to Melbourne's research publications}

Minerva Access is the Institutional Repository of The University of Melbourne

Author/s:

Heijmans, SHJ;Postoyan, R;Nesic, D;Heemels, WPMH

Title:

Reverse Average Dwell-Times for Networked Control Systems

Date:

2020-03-12

Citation:

Heijmans, S. H. J., Postoyan, R., Nesic, D. \& Heemels, W. P. M. H. (2020). Reverse

Average Dwell-Times for Networked Control Systems. Proceedings of the 2019 IEEE 58th

Conference on Decision and Control (CDC), 2019-December, pp.5480-5485. IEEE. https:// doi.org/10.1109/CDC40024.2019.9028864.

Persistent Link:

http://hdl.handle.net/11343/249532 